\title{
Release and Formation of Oxidation Related Aldehydes during Wine Oxidation
}

\author{
Mónica Bueno a , Vanesa Carrascón ${ }^{\text {a }}$, Vicente Ferreira ${ }^{* a}$ \\ ${ }^{a}$ Laboratorio de Análisis del Aroma y Enología (LAAE). Instituto Agroalimentario de Aragón \\ (IA2), Department of Analytical Chemistry, Faculty of Sciences, Universidad de Zaragoza, \\ 50009, Zaragoza, Spain
}

* To whom correspondence should be addressed

Phone: 34976762067

Fax: 34976761292

Email: vferre@unizar.es 
Table S1. Wines analyzed in the experiment including origin, age, varietal composition,

some basic compositional parameters and duration of the experiment (days).

\begin{tabular}{|c|c|c|c|c|c|c|c|c|c|}
\hline Wine & $\begin{array}{l}\text { Denomination } \\
\text { of origin }\end{array}$ & $\begin{array}{l}\text { Vintage } \\
\text { year }\end{array}$ & Grape variety & $\begin{array}{l}\text { Oak } \\
\text { aging } \\
\text { (months) }\end{array}$ & $\begin{array}{l}\text { Alcohol } \\
\%(v / v)\end{array}$ & pH & TPI $^{\mathbf{a}}$ & $\mathbf{C I}^{\mathbf{b}}$ & Days \\
\hline \multicolumn{10}{|c|}{ Red wines } \\
\hline $\mathrm{FG}$ & Campo de Borja & 2010 & Garnacha & 10 & 14.5 & 3.47 & 86.47 & 13.84 & 34.7 \\
\hline $\mathrm{TP}$ & Campo de Borja & 2011 & Garnacha & 5 & 15 & 3.42 & 57.87 & 11.21 & 29.1 \\
\hline VV & Somontano & 2012 & $\begin{array}{l}\text { Cabernet-Sauvignon, } \\
\text { Merlot }\end{array}$ & 0 & 13.5 & 3.53 & 60.90 & 10.30 & 36.5 \\
\hline $\mathrm{CH}$ & Campo de Borja & 2008 & Garnacha & 15 & 13.5 & 3.53 & 61.90 & 11.80 & 34.5 \\
\hline VN & Campo de Borja & 2010 & Garnacha & 17 & 15.5 & 3.40 & 57.90 & 13.65 & 36.3 \\
\hline UB & Cariñena & 2009 & $\begin{array}{l}\text { Syrah, Merlot, } \\
\text { Cabernet-Sauvignon }\end{array}$ & 9 & 14.5 & 3.56 & 62.33 & 11.40 & 35.5 \\
\hline $\mathrm{MZ}$ & Calatayud & 2012 & $\begin{array}{l}\text { Garnacha, } \\
\text { Tempranillo }\end{array}$ & 0 & 14 & 3.76 & 62.20 & 9.680 & 35.8 \\
\hline $\mathrm{TZ}$ & Campo de Borja & 2008 & Garnacha & 10 & 15 & 3.46 & 72.33 & 13.82 & 26.1 \\
\hline $\mathrm{RD}$ & $\begin{array}{l}\text { Vinos de la tierra } \\
\text { de Castilla }\end{array}$ & 2012 & Syrah & 0 & 14.5 & 3.74 & 69.00 & 15.47 & 33.5 \\
\hline $\mathrm{PC}$ & Ribera del Duero & 2012 & Tempranillo & 5 & 13.5 & 3.67 & 60.80 & 12.74 & 33.4 \\
\hline SL & Rioja & 2010 & $\begin{array}{l}\text { Tempranillo, } \\
\text { Mazuela, Graciano }\end{array}$ & 12 & 13.5 & 3.52 & 52.20 & 11.38 & 27.4 \\
\hline LH & Rioja & 2012 & Tempranillo & 3 & 13.5 & 3.84 & 53.40 & 8.930 & 8.1 \\
\hline TS & Toro & 2010 & Tempranillo & 14 & 14.5 & 3.91 & 66.00 & 10.29 & 27.2 \\
\hline $\mathrm{BL}$ & Rioja & 2007 & Tempranillo & 20 & 13.5 & 3.77 & 55.10 & 10.03 & 27.5 \\
\hline $\mathrm{MF}$ & Rioja & 2012 & Tempranillo & 0 & 13 & 3.93 & 48.20 & 10.64 & 27.5 \\
\hline AA & Ribera del Duero & 2008 & Tempranillo & 18 & 14.1 & 3.89 & 53.53 & 9.277 & 33.6 \\
\hline \multicolumn{10}{|c|}{ Rosé wines } \\
\hline IL & Rioja & 2012 & Garnacha & 0 & 13.5 & 3.27 & 12.75 & 1.121 & 35.3 \\
\hline EN & Somontano & 2012 & Cabernet-Sauvignon & 0 & 13.5 & 3.23 & 14.00 & 1.496 & 35.4 \\
\hline HJ & Navarra & 2012 & Garnacha & 0 & 13 & 3.19 & 11.45 & 1.180 & 36.2 \\
\hline \multicolumn{10}{|c|}{ White wines } \\
\hline VT & Rueda & 2012 & Verdejo & 0 & 12 & 3.28 & 7.57 & 0.089 & 34.2 \\
\hline MR & Rueda & 2012 & Verdejo & 0 & 13 & 3.29 & 10.15 & 0.132 & 34.5 \\
\hline FR & Rías Baixas & 2012 & Albariño & 0 & 12.5 & 3.27 & 11.48 & 0.162 & 35.0 \\
\hline $\mathrm{CC}$ & Cariñena & 2012 & Chardonnay & 0 & 13.5 & 3.48 & 11.66 & 0.152 & 35.1 \\
\hline $\mathrm{AB}$ & Calatayud & 2012 & Macabeo & 0 & 14 & 3.31 & 10.59 & 0.274 & 34.5 \\
\hline
\end{tabular}

${ }^{a}$ Total polyphenol index, expressed in absorbance x 100 in reds, x 50 in rosés and x 20

in whites. ${ }^{\mathrm{b}}$ Color index as $\left(\mathrm{A}_{420}+\mathrm{A}_{520}+\mathrm{A}_{620}\right)$ 
Table S2. Free (determined) and total (estimated) forms of wine carbonyls $(\mu \mathrm{g} / \mathrm{L})$ in the 24 wines and free $\mathrm{SO}_{2}$ content $(\mathrm{mg} / \mathrm{L})$.

\begin{tabular}{|c|c|c|c|c|c|c|c|c|c|c|c|c|}
\hline \multirow{3}{*}{ Compounds } & \multicolumn{12}{|c|}{ Red wines } \\
\hline & \multicolumn{3}{|c|}{ FG } & \multicolumn{3}{|c|}{ TP } & \multicolumn{3}{|c|}{$\mathbf{V V}$} & \multicolumn{3}{|c|}{ CH } \\
\hline & \multicolumn{2}{|c|}{ Free } & \multirow{2}{*}{$\begin{array}{c}\text { Estimated } \\
97.1\end{array}$} & \multicolumn{2}{|c|}{ Free } & \multirow{2}{*}{$\begin{array}{c}\text { Estimated } \\
60.4\end{array}$} & \multicolumn{2}{|c|}{ Free } & \multirow{2}{*}{$\begin{array}{c}\text { Estimated } \\
37.2\end{array}$} & \multicolumn{2}{|c|}{ Free } & \multirow{2}{*}{$\begin{array}{c}\text { Estimatec } \\
102\end{array}$} \\
\hline isobutyraldehyde & 37.7 & \pm 2.2 & & 21.4 & $\pm \quad 1.1$ & & 12.3 & $\pm \quad 0.5$ & & 40.1 & $\pm \quad 2.3$ & \\
\hline 2-methylbutanal & 22.1 & \pm 0.8 & 56.9 & 6.92 & \pm 0.26 & 19.5 & 4.24 & \pm 0.18 & 12.8 & 12.0 & \pm 0.5 & 30.7 \\
\hline isovaleraldehyde & 31.6 & $\pm \quad 1.1$ & 204 & 8.09 & \pm 0.30 & 69.1 & 9.5 & \pm 0.34 & 121 & 19.4 & \pm 0.7 & 120 \\
\hline decanal $^{\mathrm{a}}$ & 0.34 & \pm 0.06 & 11.9 & 0.38 & \pm 0.06 & 17.5 & 0.36 & \pm 0.06 & 27.0 & 0.33 & \pm 0.06 & 10.4 \\
\hline methional & 12.6 & $\pm \quad 0.3$ & 59.4 & 5.67 & \pm 0.18 & 27.7 & 7.39 & \pm 0.22 & 47.6 & 7.57 & \pm 0.22 & 37.3 \\
\hline phenylacetaldehyde & 33.8 & \pm 0.8 & 143 & 40.3 & $\pm \quad 0.9$ & 205 & 7.23 & \pm 0.19 & 47.0 & 27.1 & $\pm \quad 0.6$ & 101 \\
\hline benzaldehyde & 3.43 & \pm 0.29 & 8.84 & 3.46 & \pm 0.29 & 9.74 & 5.27 & \pm 0.28 & 15.9 & 3.40 & \pm 0.29 & 8.69 \\
\hline diacetyl & 948 & \pm 13 & 2443 & 2207 & $\pm \quad 35$ & 6218 & 2807 & $\pm \quad 46$ & 8480 & 697 & $\pm \quad 9$ & 1779 \\
\hline 2,3-pentanedione & 33.4 & $\pm \quad 2.4$ & 86.1 & 95.1 & $\pm \quad 7.3$ & 268 & 117 & \pm 9 & 353 & 15.9 & $\pm \quad 1.0$ & 40.7 \\
\hline furfural & 728 & \pm 26 & 1245 & 1470 & 53 & 2694 & 101 & \pm & 175 & 512 & $\pm \quad 18$ & 882 \\
\hline 5-methylfurfural & 199 & $\pm \quad 7$ & 340 & 518 & $\pm \quad 19$ & 949 & 8.63 & \pm 0.34 & 14.9 & 14.0 & \pm 0.5 & 24.1 \\
\hline$\beta$-damascenone & 2.90 & \pm 0.05 & - & 4.31 & \pm 0.08 & - & 4.41 & \pm 0.08 & - & 2.11 & \pm 0.04 & - \\
\hline$\beta$-ionone & 0.12 & \pm 0.01 & - & 0.20 & \pm 0.01 & - & 0.32 & \pm 0.01 & - & 0.04 & \pm 0.01 & - \\
\hline acetovanillone & 1398 & \pm 111 & - & 904 & $\pm \quad 70$ & - & 972 & $\pm \quad 76$ & - & 1276 & \pm 101 & - \\
\hline acetaldehyde $^{a}$ & 2261 & & 166930 & $<500$ & & $>49746$ & $<500$ & & $>81499$ & 1892 & & 125556 \\
\hline Free $\mathrm{SO}_{2}$ & 10 & & & 13 & & & 21 & & & 8.6 & & \\
\hline
\end{tabular}


Table S2 continue.

\begin{tabular}{|c|c|c|c|c|c|c|c|c|c|c|c|c|c|c|c|}
\hline \multirow{3}{*}{ Compounds } & \multicolumn{15}{|c|}{ Red wines } \\
\hline & \multicolumn{4}{|c|}{$\mathbf{V N}$} & \multicolumn{4}{|c|}{ UB } & \multicolumn{3}{|c|}{ MZ } & \multicolumn{4}{|c|}{ TZ } \\
\hline & \multicolumn{3}{|c|}{ Free } & $\begin{array}{c}\text { Estimated } \\
94.3\end{array}$ & \multicolumn{3}{|c|}{ Free } & $\begin{array}{c}\text { Estimated } \\
61.6\end{array}$ & \multicolumn{2}{|c|}{ Free } & $\begin{array}{c}\text { Estimated } \\
18.0\end{array}$ & \multicolumn{3}{|c|}{ Free } & $\begin{array}{c}\text { Estimated } \\
98.4\end{array}$ \\
\hline 2-methylbutanal & 10.9 & \pm & 0.4 & 32.9 & 5.98 & \pm & 0.23 & 19.8 & 3.19 & \pm 0.15 & 7.67 & 11.0 & \pm & 0.4 & 26.6 \\
\hline isovaleraldehyde & 8.27 & \pm & 0.31 & 88.7 & 7.54 & \pm & 0.30 & 122 & 4.42 & \pm 0.27 & 33.1 & 4.96 & \pm & 0.27 & 20.2 \\
\hline methional & 6.06 & \pm & 0.19 & 37.7 & 6.01 & \pm & 0.19 & 48.8 & 4.83 & \pm 0.17 & 23.0 & 6.16 & \pm & 0.19 & 17.6 \\
\hline phenylacetaldehyde & 30.2 & \pm & 0.7 & 196 & 18.9 & \pm & 0.4 & 165 & 4.83 & \pm 0.16 & 19 & 38.6 & \pm & 0.9 & 88.6 \\
\hline benzaldehyde & 8.59 & \pm & 0.28 & 25.9 & 4.02 & \pm & 0.28 & 13.3 & 13.8 & \pm 0.3 & 33 & 3.94 & \pm & 0.28 & 9.51 \\
\hline diacetyl & 4829 & \pm & 82 & 14543 & 4429 & \pm & 74 & 14648 & 3466 & 57 & 8333 & 483 & \pm & 7 & 1165 \\
\hline 2,3-pentanedione & 90.1 & \pm & 6.9 & 271 & 123 & \pm & 10 & 407 & 99 & 8 & 237 & 12.2 & \pm & 0.7 & 29.4 \\
\hline$\beta$-damascenone & 3.23 & \pm & 0.06 & - & 1.76 & \pm & 0.03 & - & 6.54 & \pm 0.13 & - & 2.79 & \pm & 0.05 & - \\
\hline$\beta$-ionone & 0.32 & \pm & 0.01 & - & 0.07 & \pm & 0.01 & - & 0.17 & \pm 0.01 & - & 0.12 & \pm & 0.01 & - \\
\hline acetovanillone & 1463 & \pm & 117 & - & 1082 & \pm & 85 & - & 520 & 38 & - & 1495 & \pm & 119 & - \\
\hline acetaldehyde $^{\mathrm{a}}$ & 751 & & & 90238 & $<500$ & & & $>93948$ & 911 & & 99807 & 996 & & & 36195 \\
\hline Free $\mathrm{SO}_{2}$ & 16 & & & & 25 & & & & 14 & & & 4.7 & & & \\
\hline
\end{tabular}


Table S2 continue.

\begin{tabular}{|c|c|c|c|c|c|c|c|c|c|c|c|c|}
\hline \multirow{3}{*}{ Compounds } & \multicolumn{12}{|c|}{ Red wines } \\
\hline & \multicolumn{3}{|c|}{ RD } & \multicolumn{3}{|c|}{ PC } & \multicolumn{3}{|c|}{ SL } & \multicolumn{3}{|c|}{ LH } \\
\hline & \multicolumn{2}{|c|}{ Free } & \multirow{2}{*}{$\frac{\text { Estimated }}{14.1}$} & \multicolumn{2}{|c|}{ Free } & \multirow{2}{*}{$\begin{array}{c}\text { Estimated } \\
19.8\end{array}$} & \multicolumn{2}{|c|}{ Free } & \multirow{2}{*}{$\begin{array}{c}\text { Estimated } \\
30.4\end{array}$} & \multicolumn{2}{|c|}{ Free } & \multirow{2}{*}{$\frac{\text { Estimated }}{20.7}$} \\
\hline isobutyraldehyde & 7.22 & \pm 0.21 & & 7.71 & \pm 0.24 & & 10.6 & $\pm \quad 0.4$ & & 8.40 & \pm 0.29 & \\
\hline 2-methylbutanal & 3.61 & \pm 0.16 & 7.02 & 2.74 & \pm 0.15 & 7.04 & 4.20 & \pm 0.18 & 12.0 & 3.91 & \pm 0.17 & 9.66 \\
\hline isovaleraldehyde & 15.1 & $\pm \quad 0.5$ & 38.6 & 8.46 & \pm 0.31 & 112 & 8.20 & \pm 0.31 & 113 & 6.68 & \pm 0.28 & 81.5 \\
\hline decanal $^{\mathrm{a}}$ & 0.81 & \pm 0.05 & 16.1 & 0.64 & \pm 0.05 & 89.8 & 1.11 & \pm 0.04 & 122 & 0.41 & \pm 0.06 & 41.8 \\
\hline methional & 11.4 & $\pm \quad 0.3$ & 24.3 & 7.03 & \pm 0.21 & 58.9 & 7.57 & \pm 0.22 & 64.3 & 6.87 & \pm 0.20 & 51.0 \\
\hline phenylacetaldehyde & 12.0 & \pm 0.3 & 17.9 & 7.63 & \pm 0.20 & 59.6 & 11.2 & \pm 0.3 & 93.7 & 9.19 & \pm 0.23 & 64.4 \\
\hline benzaldehyde & 5.07 & \pm 0.28 & 9.88 & 4.38 & \pm 0.28 & 11.3 & 6.43 & \pm 0.28 & 18.4 & 11.5 & \pm 0.3 & 28.4 \\
\hline diacetyl & 3090 & $\pm \quad 51$ & 6018 & 1565 & $\pm \quad 24$ & 4027 & 2060 & $\pm \quad 32$ & 5898 & 1530 & \pm & 3775 \\
\hline 2,3-pentanedione & 212 & $\pm \quad 17$ & 413 & 60.4 & \pm 4.5 & 156 & 76.9 & $\pm \quad 5.8$ & 220 & 60.2 & \pm 4.5 & 149 \\
\hline furfural & 103 & $\pm \quad 4$ & 169 & 129 & $\pm \quad 5$ & 207 & 198 & $\pm \quad 7$ & 322 & 151 & $\pm \quad 5$ & 240 \\
\hline 5-methylfurfural & 3.14 & \pm 0.18 & 5.14 & 57.8 & $\pm \quad 2.1$ & 92.8 & 8.37 & \pm 0.34 & 13.6 & 3.27 & \pm 0.19 & 5.20 \\
\hline$\beta$-damascenone & 5.17 & \pm 0.10 & - & 2.93 & \pm 0.05 & - & 1.99 & \pm 0.03 & - & 2.70 & \pm 0.05 & - \\
\hline$\beta$-ionone & 0.40 & \pm 0.01 & - & 0.34 & \pm 0.01 & - & 0.21 & \pm 0.01 & - & 0.21 & \pm 0.01 & - \\
\hline acetovanillone & 309 & $\pm \quad 21$ & - & 228 & \pm 14 & - & 294 & $\pm \quad 20$ & - & 90.5 & \pm 5.8 & - \\
\hline acetaldehyde $^{\mathrm{a}}$ & 5873 & & 246211 & 833 & & 253564 & 1762 & & 419909 & $<500$ & & $>110989$ \\
\hline Free $\mathrm{SO}_{2}$ & 5.4 & & & 40 & & & 31 & & & 29 & & \\
\hline
\end{tabular}


Table S2 continue.

\begin{tabular}{|c|c|c|c|c|c|c|c|c|c|c|c|c|}
\hline \multirow{3}{*}{ Compounds } & \multicolumn{12}{|c|}{ Red wines } \\
\hline & \multicolumn{3}{|c|}{ TS } & \multicolumn{3}{|c|}{ BL } & \multicolumn{3}{|c|}{ MF } & \multicolumn{3}{|c|}{ AA } \\
\hline & \multicolumn{2}{|c|}{ Free } & \multirow{2}{*}{$\frac{\text { Estimated }}{21.2}$} & \multicolumn{2}{|c|}{ Free } & \multirow{2}{*}{$\begin{array}{c}\text { Estimated } \\
46.9\end{array}$} & \multicolumn{2}{|c|}{ Free } & \multirow{2}{*}{$\begin{array}{c}\text { Estimated } \\
9.22\end{array}$} & \multicolumn{2}{|c|}{ Free } & \multirow{2}{*}{$\begin{array}{c}\text { Estimated } \\
23.2\end{array}$} \\
\hline isobutyraldehyde & 8.56 & \pm 0.30 & & 20.5 & $\pm \quad 1.1$ & & 4.42 & \pm 0.03 & & 9.48 & \pm 0.35 & \\
\hline 2-methylbutanal & 4.30 & \pm 0.18 & 10.7 & 6.73 & \pm 0.26 & 15.4 & 3.24 & \pm 0.15 & 6.77 & 3.73 & \pm 0.17 & 9.12 \\
\hline isovaleraldehyde & 7.43 & \pm 0.29 & 92.1 & 12.3 & $\pm \quad 0.4$ & 122 & 10.5 & $\pm \quad 0.4$ & 106 & 10.5 & $\pm \quad 0.4$ & 142 \\
\hline decanal $^{\mathrm{a}}$ & 0.74 & \pm 0.05 & 96.6 & 1.08 & \pm 0.04 & 68.9 & 0.86 & \pm 0.05 & 121 & 0.82 & \pm 0.05 & 58.2 \\
\hline methional & 7.45 & \pm 0.22 & 62.9 & 8.58 & \pm 0.24 & 62.2 & 8.38 & \pm 0.24 & 60.1 & 5.59 & \pm 0.18 & 43.8 \\
\hline phenylacetaldehyde & 19.5 & \pm 0.4 & 154 & 17.5 & \pm 0.4 & 98.4 & 9.98 & \pm 0.24 & 58.0 & 11.9 & \pm 0.3 & 85.9 \\
\hline benzaldehyde & 4.59 & \pm 0.28 & 11.4 & 7.36 & \pm 0.28 & 16.9 & 6.81 & \pm 0.28 & 14.2 & 4.55 & \pm 0.28 & 11.1 \\
\hline diacetyl & 1193 & $\pm \quad 17$ & 2955 & 840 & $\pm \quad 12$ & 1923 & 2549 & $\pm \quad 41$ & 5319 & 359 & \pm & 878 \\
\hline 2,3-pentanedione & 33.7 & $\pm \quad 2.4$ & 83.6 & 17.3 & \pm 1.1 & 39.7 & 135 & $\pm \quad 10$ & 281 & 9.99 & \pm 0.5 & 24.4 \\
\hline furfural & 266 & $\pm \quad 10$ & 424 & 489 & $\pm \quad 18$ & 748 & 74.8 & \pm 2.6 & 120 & 81.6 & \pm 2.9 & 133 \\
\hline 5-methylfurfural & 46.2 & \pm 1.7 & 73.4 & 23.1 & $\pm \quad 0.9$ & 35.3 & 0.25 & \pm 0.15 & 0.40 & 24.3 & \pm 0.9 & 39.6 \\
\hline$\beta$-damascenone & 0.81 & \pm 0.01 & - & 1.10 & \pm 0.01 & - & 6.89 & \pm 0.14 & - & 1.17 & \pm 0.02 & - \\
\hline$\beta$-ionone & 0.16 & \pm 0.01 & - & 0.12 & \pm 0.01 & - & 0.28 & \pm 0.01 & - & 0.12 & \pm 0.01 & - \\
\hline acetovanillone & 198 & 12 & - & 500 & $\pm \quad 37$ & - & 261 & $\pm \quad 17$ & - & 252 & \pm 16 & - \\
\hline acetaldehyde $^{\mathrm{a}}$ & 1462 & & 411509 & 653 & & 89669 & $<500$ & & $>152062$ & 2215 & & 337785 \\
\hline Free $\mathrm{SO}_{2}$ & 37 & & & 18 & & & 40 & & & 20 & & \\
\hline
\end{tabular}


Table S2 continue.

\begin{tabular}{|c|c|c|c|c|c|c|c|c|c|c|}
\hline \multirow{3}{*}{ Compounds } & \multicolumn{10}{|c|}{ Rosé wines } \\
\hline & \multicolumn{4}{|c|}{ IL } & \multicolumn{3}{|c|}{ EN } & \multicolumn{3}{|c|}{ HJ } \\
\hline & \multicolumn{3}{|c|}{ Free } & \multirow{2}{*}{$\begin{array}{c}\text { Estimated } \\
27.0\end{array}$} & \multicolumn{2}{|c|}{ Free } & \multirow{2}{*}{$\begin{array}{c}\text { Estimated } \\
27.4\end{array}$} & \multicolumn{2}{|c|}{ Free } & \multirow{2}{*}{$\begin{array}{c}\text { Estimated } \\
29.3\end{array}$} \\
\hline isobutyraldehyde & 8.75 & \pm & 0.31 & & 7.73 & \pm 0.24 & & 12.4 & 0.5 & \\
\hline 2-methylbutanal & 3.03 & \pm & 0.15 & 9.35 & 2.14 & \pm 0.14 & 7.58 & 2.88 & \pm 0.15 & 6.79 \\
\hline isovaleraldehyde & 8.77 & \pm & 0.32 & 118 & 5.21 & \pm 0.27 & 79.4 & 14.7 & 0.5 & 41.8 \\
\hline decanal $^{\mathrm{a}}$ & 1.47 & \pm & 0.04 & 111 & 0.97 & \pm 0.04 & 78.4 & 1.02 & \pm 0.04 & 17.3 \\
\hline methional & 3.53 & \pm & 0.15 & 26.4 & 4.67 & \pm 0.16 & 43.6 & 3.87 & 0.15 & 9.92 \\
\hline phenylacetaldehyde & 2.63 & \pm & 0.14 & 20.8 & 9.09 & \pm 0.23 & 86.9 & 5.99 & 0.17 & 10.0 \\
\hline benzaldehyde & 13.3 & \pm & 0.3 & 41.0 & 2.56 & \pm 0.29 & 9.06 & 6.56 & \pm 0.28 & 15.5 \\
\hline diacetyl & 505 & \pm & 7 & 1558 & 632 & \pm & 2239 & 1040 & 15 & 2455 \\
\hline 2,3-pentanedione & 49.7 & \pm & 3.7 & 153 & 88.3 & $\pm \quad 6.7$ & 313 & 69.6 & 5.3 & 164 \\
\hline furfural & 79.3 & \pm & 2.8 & 131 & 92.9 & 3.3 & 142 & 170 & \pm & 266 \\
\hline 5-methylfurfural & 2.25 & \pm & 0.17 & 3.72 & 4.60 & \pm 0.22 & 7.02 & 5.79 & \pm 0.25 & 9.08 \\
\hline$\beta$-damascenone & 10.3 & \pm & 0.2 & - & 11.8 & 0.2 & - & 17.8 & 0.4 & - \\
\hline$\beta$-ionone & 0.07 & \pm & 0.01 & - & 0.16 & \pm 0.01 & - & 0.04 & \pm 0.01 & - \\
\hline acetovanillone & 665 & \pm & 50 & - & 1285 & \pm 102 & - & 822 & 63 & - \\
\hline acetaldehyde $^{\mathrm{a}}$ & $<500$ & & & $>81321$ & $<500$ & & $>86550$ & 7890 & & 282316 \\
\hline Free $\mathrm{SO}_{2}$ & 21 & & & & 23 & & & 4.6 & & \\
\hline
\end{tabular}


Table S2 continue.

\begin{tabular}{|c|c|c|c|c|c|c|c|c|c|c|c|c|c|c|c|c|c|c|c|}
\hline \multirow{4}{*}{$\begin{array}{c}\text { Compounds } \\
\text { isobutyraldehyde }\end{array}$} & \multicolumn{19}{|c|}{ White wines } \\
\hline & \multicolumn{4}{|c|}{ VT } & \multicolumn{4}{|c|}{ MR } & \multicolumn{3}{|c|}{ FR } & \multicolumn{4}{|c|}{$\mathrm{CC}$} & \multicolumn{4}{|c|}{$\mathbf{A B}$} \\
\hline & \multicolumn{3}{|c|}{ Free } & \multirow{2}{*}{$\begin{array}{c}\text { Estimated } \\
19.8\end{array}$} & \multicolumn{3}{|c|}{ Free } & \multirow{2}{*}{$\begin{array}{c}\text { Estimated } \\
19.8\end{array}$} & \multicolumn{2}{|c|}{ Free } & \multirow{2}{*}{$\begin{array}{c}\text { Estimated } \\
30.5\end{array}$} & \multicolumn{3}{|c|}{ Free } & \multirow{2}{*}{$\begin{array}{c}\text { Estimated } \\
24.8\end{array}$} & \multicolumn{3}{|c|}{ Free } & \multirow{2}{*}{$\begin{array}{c}\text { Estimated } \\
18.0\end{array}$} \\
\hline & 4.84 & \pm & 0.06 & & 5.53 & \pm & 0.10 & & 5.60 & \pm 0.11 & & 7.32 & \pm & 0.22 & & 4.44 & \pm & 0.03 & \\
\hline 2-methylbutanal & 2.30 & \pm & 0.14 & 9.39 & 1.81 & \pm & 0.13 & 6.48 & 1.55 & \pm 0.13 & 8.44 & 2.86 & \pm & 0.15 & 9.67 & 1.39 & \pm & 0.13 & 5.62 \\
\hline isovaleraldehyde & 6.37 & \pm & 0.28 & 153 & 4.15 & \pm & 0.27 & 79.1 & 4.07 & \pm 0.27 & 116 & 7.25 & \pm & 0.29 & 121 & 4.78 & \pm & 0.27 & 95.3 \\
\hline decanal $^{\mathrm{a}}$ & 1.25 & \pm & 0.04 & 129 & 1.39 & \pm & 0.04 & 161 & 1.09 & \pm 0.04 & 169 & 1.21 & \pm & 0.04 & 81.0 & 1.12 & \pm & 0.04 & 86.7 \\
\hline methional & 4.80 & \pm & 0.16 & 52.8 & 2.77 & \pm & 0.14 & 27.1 & 3.85 & \pm 0.15 & 39.7 & 4.35 & \pm & 0.16 & 41.4 & 2.39 & \pm & 0.14 & 23.6 \\
\hline phenylacetaldehyde & 13.2 & \pm & 0.3 & 185 & 3.91 & \pm & 0.15 & 45.7 & 8.03 & \pm 0.21 & 130 & 5.86 & \pm & 0.17 & 52.7 & 16.4 & \pm & 0.4 & 195 \\
\hline benzaldehyde & 92.2 & \pm & 1.7 & 376 & 62.9 & \pm & 1.1 & 225 & 17.9 & $\pm \quad 0.3$ & 97.6 & 6.09 & \pm & 0.28 & 20.6 & 34.4 & \pm & 0.6 & 139 \\
\hline diacetyl & 30.9 & \pm & 7.0 & 126 & 28.5 & \pm & 7.0 & 102 & 54.2 & $\pm \quad 6.7$ & 295 & 74.7 & \pm & 6.5 & 253 & 32.1 & \pm & 7.0 & 130 \\
\hline 2,3-pentanedione & 1.39 & \pm & 0.20 & 5.66 & 1.59 & \pm & 0.18 & 5.68 & 1.40 & \pm 0.20 & 7.62 & 2.00 & \pm & 0.15 & 6.77 & 2.65 & \pm & 0.11 & 10.7 \\
\hline furfural & 63.4 & \pm & 2.2 & 107 & 206 & \pm & 7 & 338 & 502 & $\pm \quad 18$ & 829 & 169 & \pm & 6 & 267 & 279 & \pm & 10 & 456 \\
\hline 5-methylfurfural & 2.91 & \pm & 0.18 & 4.90 & 3.79 & \pm & 0.20 & 6.22 & 8.72 & \pm 0.35 & 14.4 & 3.23 & \pm & 0.18 & 5.09 & 3.90 & \pm & 0.20 & 6.37 \\
\hline$\beta$-damascenone & 4.60 & \pm & 0.09 & - & 8.27 & \pm & 0.16 & - & 2.78 & \pm 0.05 & - & 5.83 & \pm & 0.11 & - & 5.01 & \pm & 0.10 & - \\
\hline$\beta$-ionone & 0.06 & \pm & 0.01 & - & 0.06 & \pm & 0.01 & - & 0.11 & \pm 0.01 & - & 0.05 & \pm & 0.01 & - & 0.04 & \pm & 0.01 & - \\
\hline acetovanillone & 576 & \pm & 43 & - & 996 & \pm & 78 & - & 1646 & \pm 132 & - & 456 & \pm & 33 & - & 672 & \pm & 51 & - \\
\hline acetaldehyde $^{\mathrm{a}}$ & 897 & & & 100266 & $<500$ & & & $>250789$ & $<500$ & & $>167295$ & $<500$ & & & $>71924$ & $<500$ & & & $>83405$ \\
\hline Free $\mathrm{SO}_{2}$ & 37 & & & & 26 & & & & 44 & & & 19 & & & & 22 & & & \\
\hline
\end{tabular}

${ }^{\text {a }}$ The apparent formation constant $\left(K_{a}\right)$ published by de Azevedo et al. $2007^{15}$ was used for the calculation of the estimated total amount 
Table S3. Concentration ranges and average concentrations in the initial wines of amino acids and alcohols potentially precursors for oxidation aldehydes and some trace mineral elements with potential catalytic activity upon the oxidation processes.

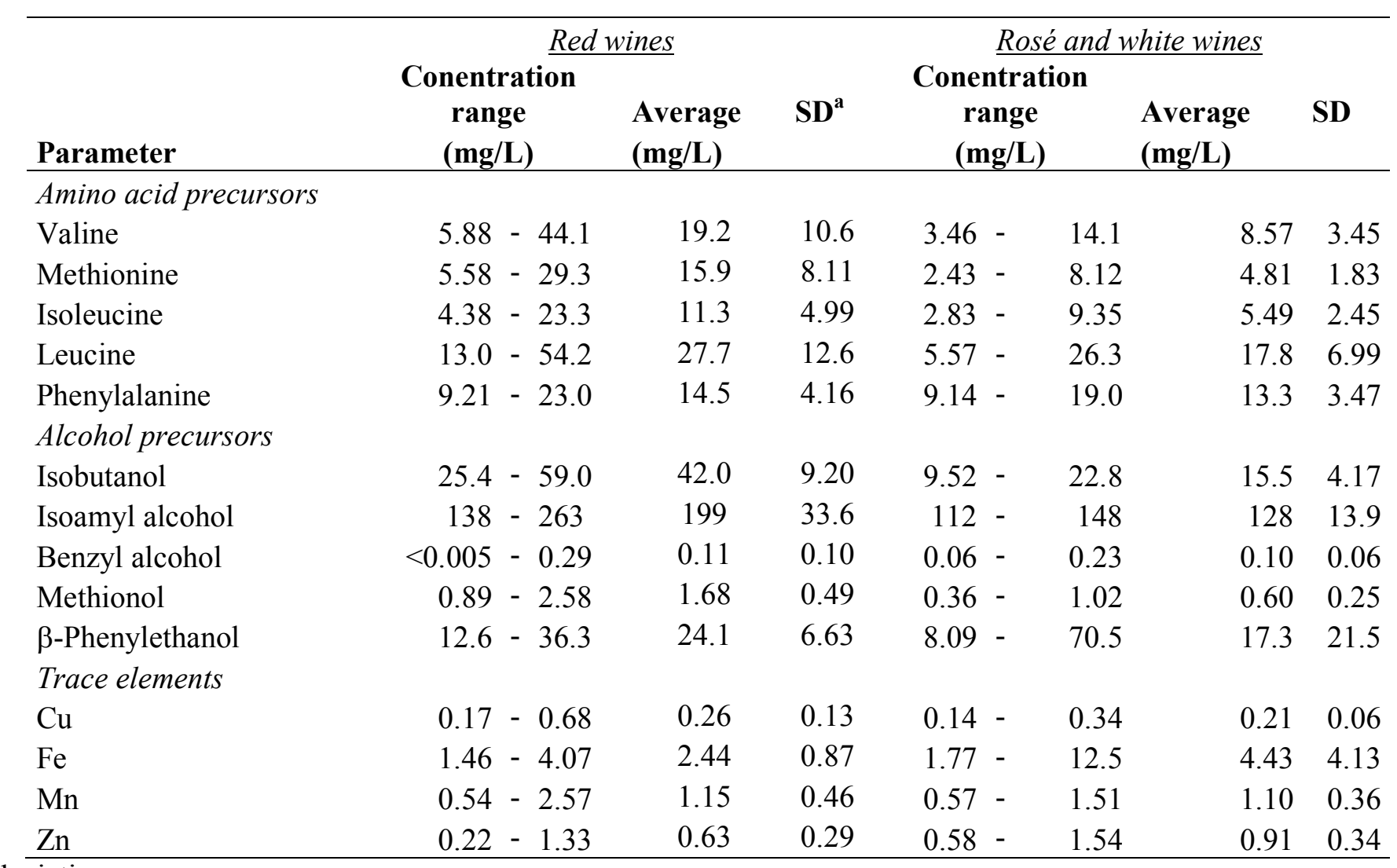

${ }^{\mathrm{a}} \mathrm{SD}$ : standard deviation 
Figure S1. Measured levels of different surrogates as a function of wine molecular $\mathrm{SO}_{2}$ content: (a) 3-(methylthio)butanal and (b) hydrocinnamaldehyde. Solid line gives the expected free level estimated using the surrogate- $\mathrm{SO}_{2}$ adduct dissociation constant measured in real wines.

a)

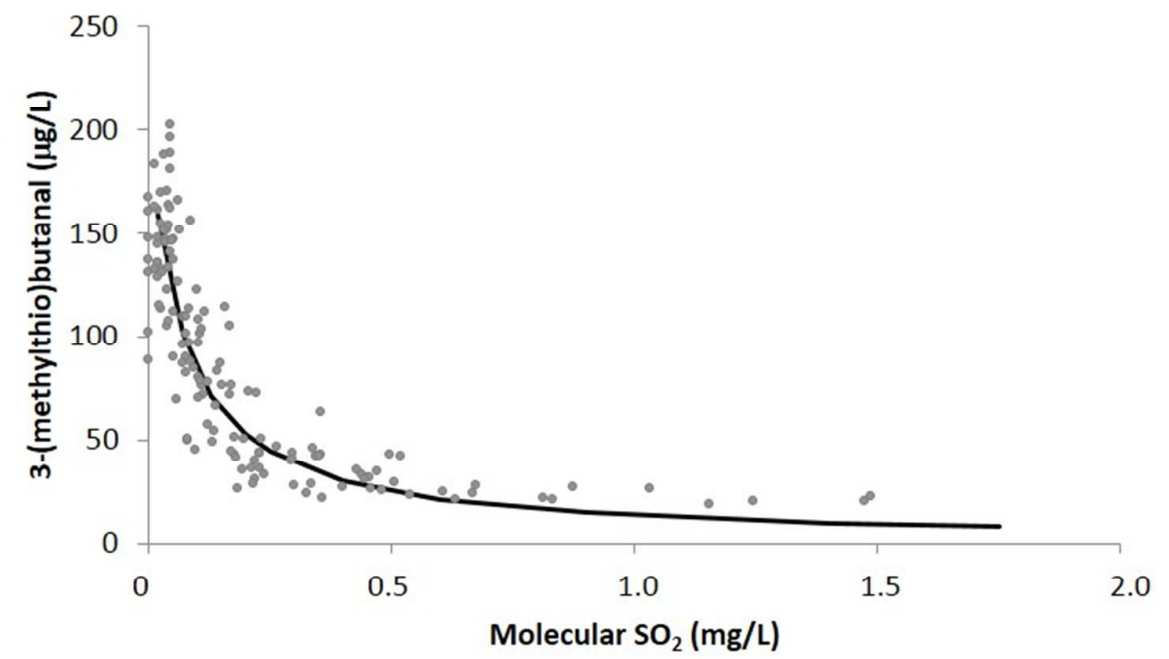

b)

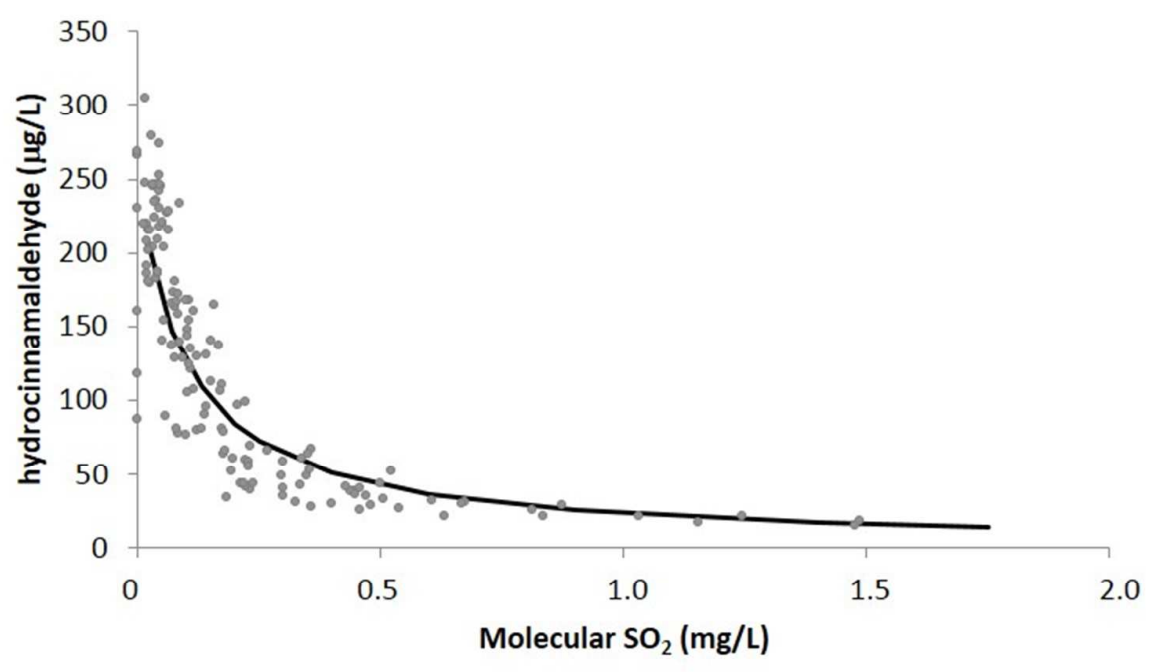


Figure S2. Relationship between the inverse of the molar concentration of bound forms (1/B) and molecular $\mathrm{SO}_{2}$. The slope represents $1 / \mathrm{K} a$ in real wines.

a) 3-(methylthio)butanal, b) hydrocinnamaldehyde, c) 3,5,5-trimethylhexanal.

a)

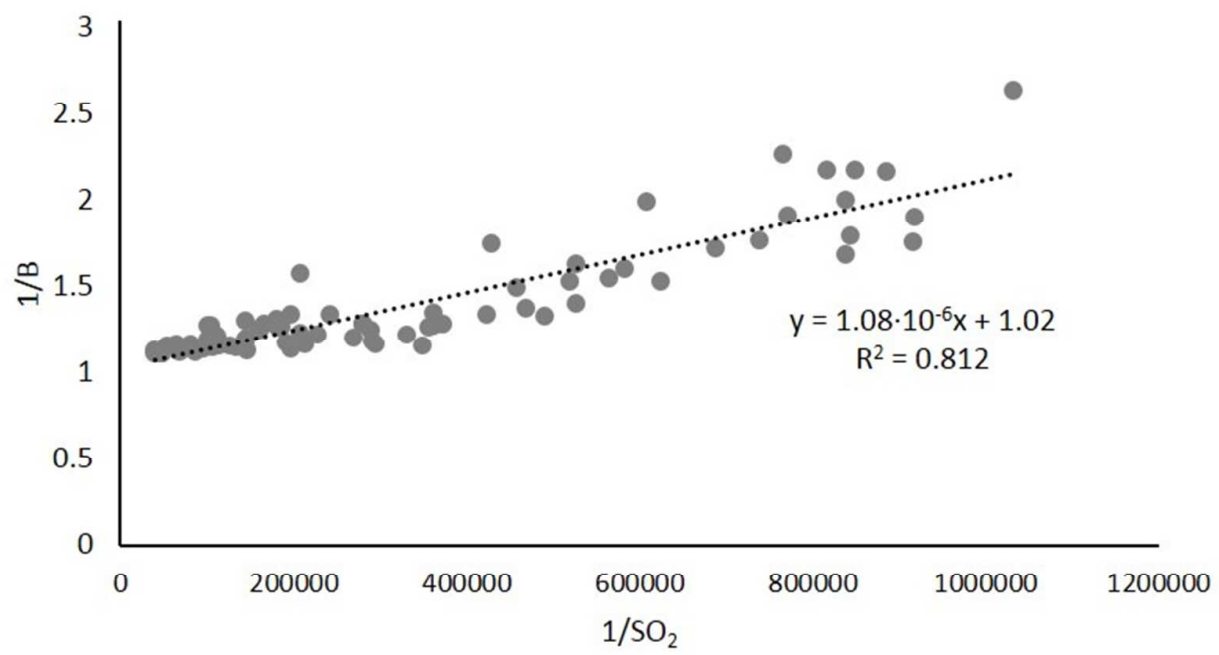

b)

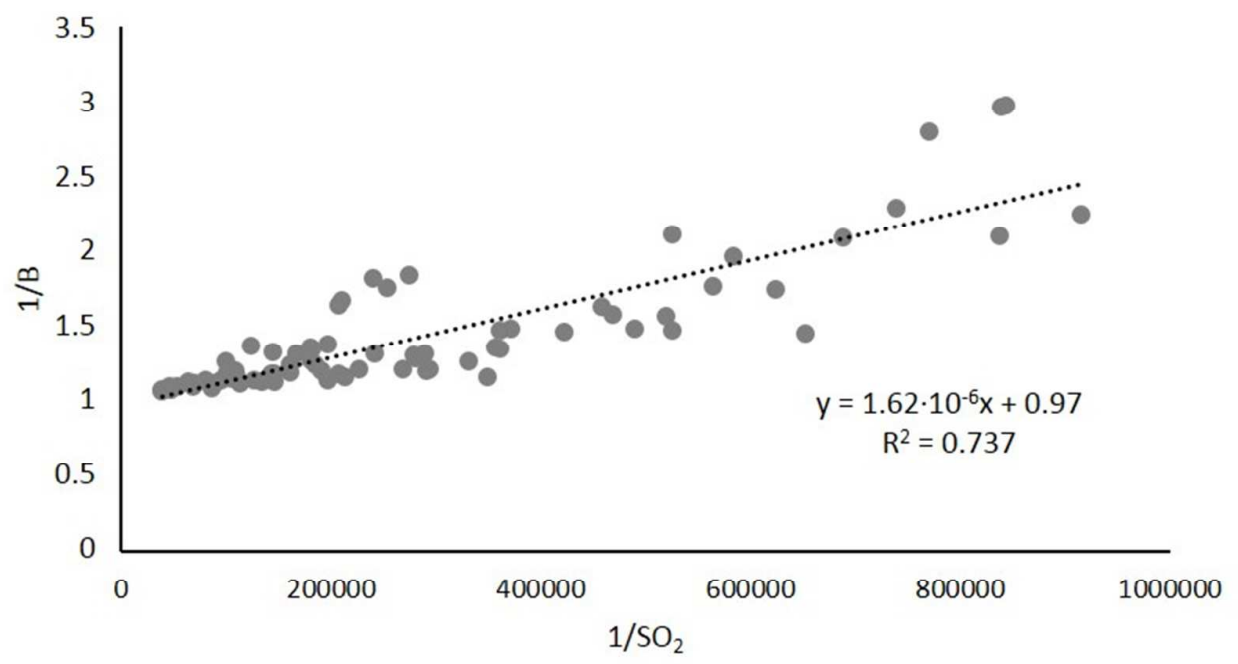


c)

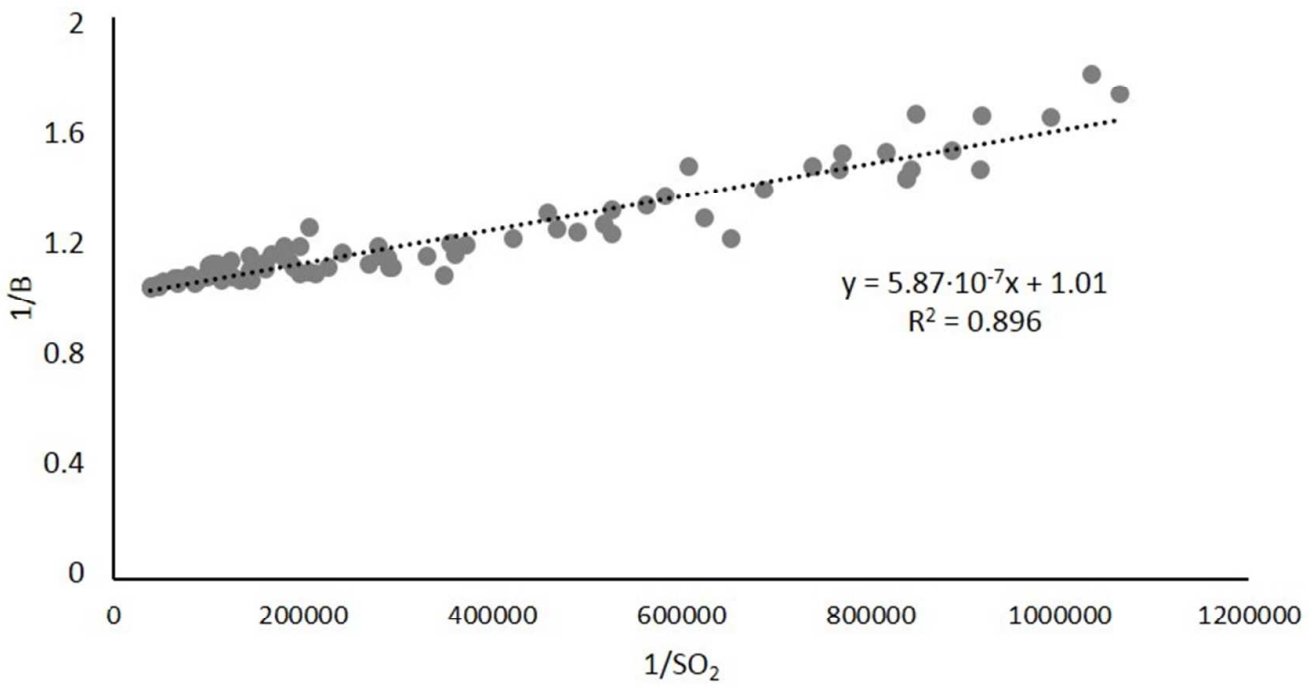


Figure S3. Differences between the measured and estimated free levels of some aldehydes during wine oxidation as a function of the molecular $\mathrm{SO}_{2}$ level of the wine. The data from 24 wines after 5 different oxidation levels are represented: (a) isobutyraldehyde. (b) isovaleraldehyde, (c) phenylacetaldehyde,

For phenylacetaldehyde and isovaleraldehyde, apparent formation constant $\left(K_{a}\right)$ for the corresponding surrogate calculated in real wine was taken. In case of isobutyraldehyde the $K_{a}$ reported in synthetic wine by Bueno et al. $2014^{19}$ was used.

a)

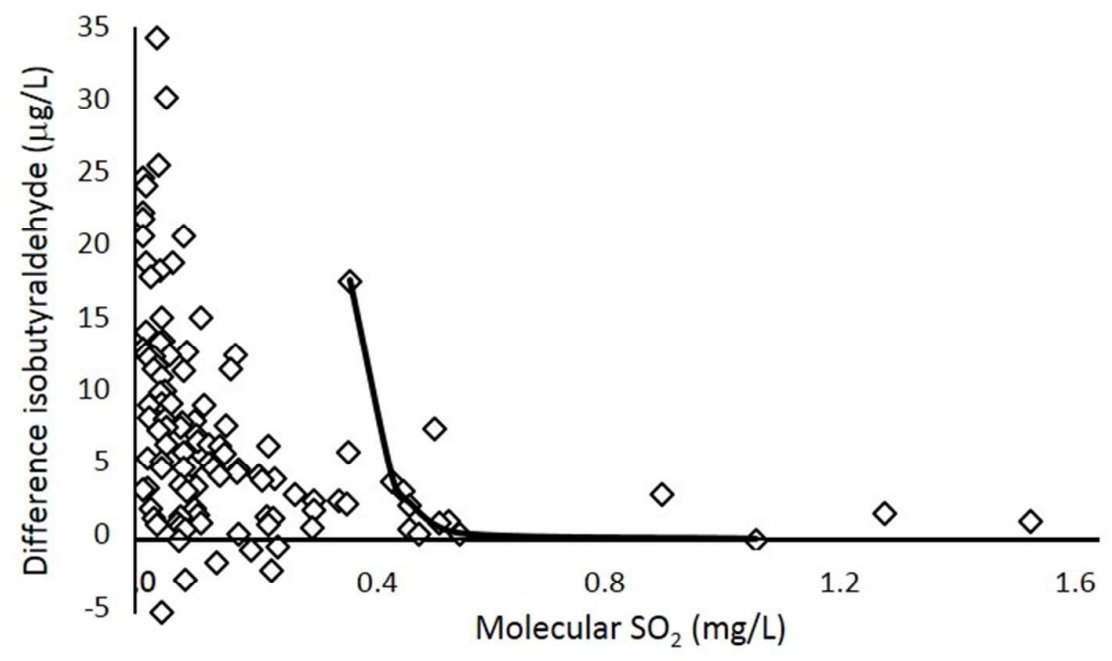

b)

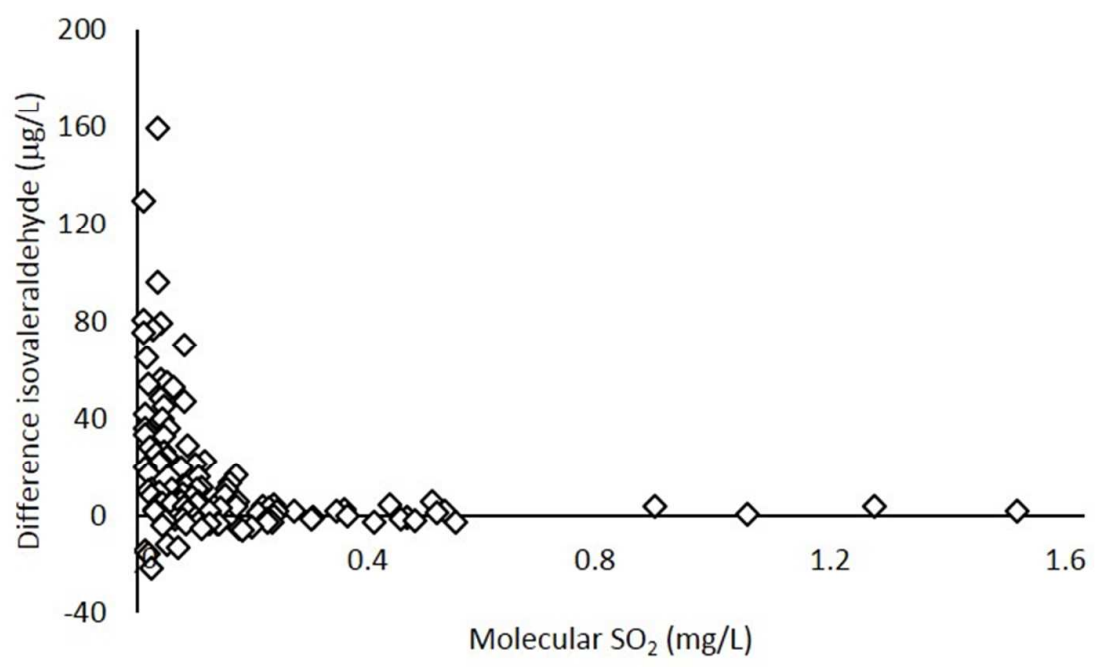


c)

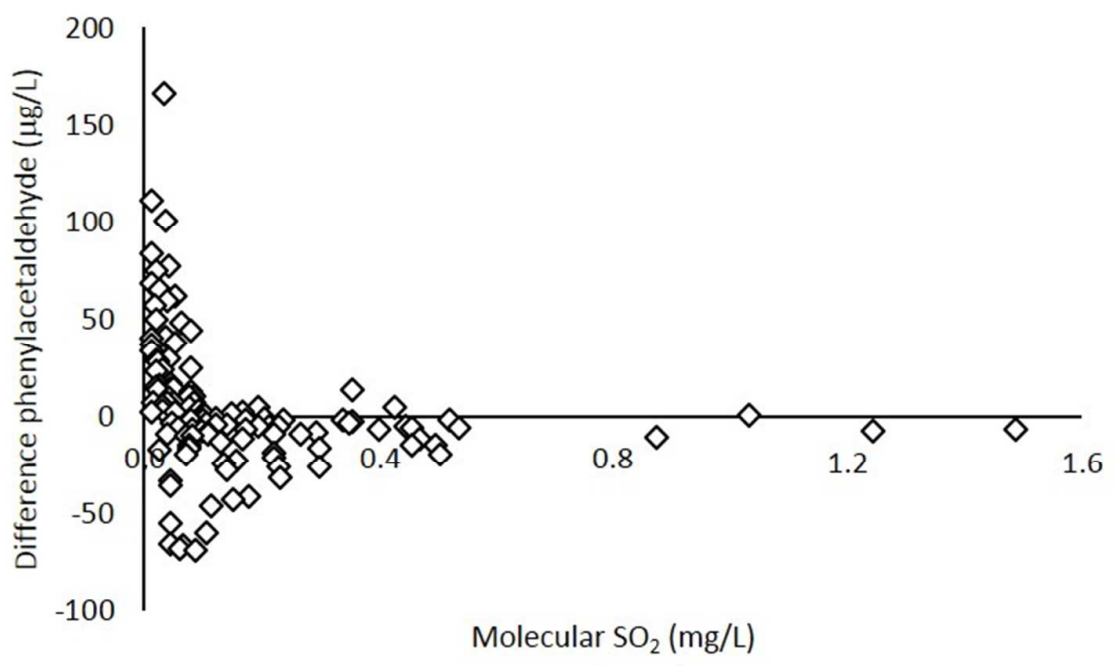

